\title{
Estado actual del PET en los tumores de cabeza y cuello: impacto en la planificación del tratamiento radioterápico
}

\section{Current state of PET in head and neck tumours: impact on the planning of radiotherapy treatment}

\author{
F. Arias de la Vega ${ }^{1}$, M.J. García-Velloso², G. Asín ${ }^{1}$, M. Rico ${ }^{1}$, M.T. Vila ${ }^{1}$, V. Chicata ${ }^{1}$
}

\section{RESUMEN}

El uso de la tomografía de emisión de positrones (PET) en los tumores de cabeza y cuello está cada vez más extendido. A sus indicaciones clínicas -especialmente en el estadiaje de los pacientes pero también en la valoración de la respuesta al tratamiento y en la detección o confirmación de recidivas-, se une ahora el posible impacto terapéutico a través de su aportación en la planificación del tratamiento radioterápico. La integración de las imágenes del PET en el proceso radioterápico parece prometedora, aunque persisten importantes dudas acerca del mismo que hacen que de momento se reserve al campo de la investigación. En este trabajo se revisa el estado actual del PET en el área de los tumores de cabeza y cuello, así como su impacto en la planificación del tratamiento radioterápico.

Palabras clave. PET. Cáncer de cabeza y cuello. Radioterapia. Planificación.

\begin{abstract}
The use of positron emission tomography (PET) in head and neck tumours is increasingly widespread. To its clinical indications -especially in the staging of patients but also in evaluating response to treatment and in detecting or confirming relapses- is now added its possible therapeutic impact through its contribution to the planning of radiotherapy treatment. The integration of PET images in the radiotherapy process seems promising, although important doubts remain about it, which means that it is still under research. This article reviews the current state of PET in the area of head and neck tumours, as well as its impact on radiotherapy treatment planning.
\end{abstract}

Key words. PET. Head and neck tumours. Radiotherapy. Planning.
1. Servicio de Oncología Radioterápica.

Hospital de Navarra. Pamplona.

2. Servicio de Medicina Nuclear.

Clínica Universidad de Navarra. Pamplona.

\author{
Correspondencia \\ Fernando Arias de la Vega \\ Servicio de Oncología Radioterápica \\ Hospital de Navarra \\ Irunlarrea, 3 \\ 31008 Pamplona \\ E-mail: farias.delavega@terra.es
}




\section{INTRODUCCIÓN}

Los tumores de cabeza y cuello incluyen los carcinomas epidermoides de la cavidad oral, faringe, laringe y los menos frecuentes de la cavidad nasal, senos paranasales y glándulas salivares. El conjunto de los cánceres de cabeza y cuello son después de los cánceres de pulmón, próstata y colorrectal los que ocupan el cuarto lugar en hombres en la Unión Europea. Concretamente en $\mathrm{Na}-$ varra la incidencia durante el quinquenio 1998-2002 fue del 48 y 6,8 por mil en varones y en mujeres, respectivamente ${ }^{1}$. A pesar de que el $80 \%$ de los tumores malignos de cabeza y cuello diagnosticados en estadios precoces pueden ser curados, casi un $70 \%$ de los pacientes tienen enfermedad localmente avanzada o metástasis ganglionares al diagnóstico, lo que conlleva un pronóstico mucho peor, con cifras de curación en torno al $30 \%{ }^{2}$. El factor pronóstico más importante en los carcinomas escamosos de cabeza y cuello es la extensión ganglionar de la enfermedad. Además, estos tumores y sus tratamientos pueden tener un gran impacto sobre funciones básicas como la alimentación, respiración o el habla y el tratamiento puede afectar de manera significativa la apariencia externa de los pacientes. El abordaje a menudo requiere colaboración multidisciplinar y las técnicas de diagnóstico por imagen desempeñan una función muy importante tanto en la estadificación inicial, como en la planificación del tratamiento, la evaluación de la respuesta y la reestadificación y seguimiento de los pacientes.

La tomografía computarizada (TC) y la resonancia magnética (RM) son las técnicas de diagnóstico convencional (TDC) en el manejo clínico de estos pacientes. Son técnicas de imagen estructural y permiten identificar cambios de tamaño y distorsión anatómica. Sin embargo, presentan algunas limitaciones tales como: sensibilidad baja para detectar enfermedad de pequeño tamaño, inferior a $1 \mathrm{~cm}$; incapacidad para proporcionar valoración precoz de la respuesta al tratamiento y dificultad para diferenciar enfermedad residual/recidiva de cambios secundarios a los tratamientos.
La tomografía por emisión de positrones (PET) es una técnica de diagnóstico por imagen de medicina nuclear que permite analizar la captación y retención de diferentes radiofármacos por las células y proporcionar información metabólica sobre procesos bioquímicos. Mejora la detección de infiltración ganglionar tumoral y de metástasis a distancia respecto a las TDC $^{3}$. Permite caracterizar si las alteraciones estructurales son debidas al tumor, a secuelas de tratamientos previos o a otros procesos biológicos. Por tanto, la PET proporciona información adicional y complementa las TDC. La mayor evidencia se da en pacientes con tumores de cabeza y cuello de estirpe escamosa y en pacientes con cáncer diferenciado de tiroides ${ }^{4}$.

Los estudios PET se realizan habitualmente con un radiofármaco análogo de la glucosa denominado $2{ }^{18} \mathrm{~F}$-fluoro-2-desoxiD-glucosa (FDG). Una vez en el interior de las células la FDG es fosforilada por la enzima hexokinasa y, a diferencia de la glucosa, queda atrapada dentro de las células sin seguir los pasos ulteriores de la cadena glicolítica, ya que esta molécula no es sustrato de la glucosa-6-fosfato isomerasa, enzima que interviene en el siguiente paso de la cadena. Tampoco puede seguir las vías metabólicas distintas a la glicolisis. Por todo ello, la captación de FDG representa la concentración de glucosa marcada y el grado de actividad glicolítica presente en el tejido. Las células tumorales en general y el carcinoma escamoso especialmente presentan un metabolismo de glucosa aumentado con relación al tejido sano adyacente. Esta mayor avidez por la FDG, mediada por los transportadores de glucosa celulares, permite obtener una gran resolución de contraste en las imágenes PET. Las lesiones tumorales se presentan como imágenes hipercaptantes o hipermetabólicas.

La intensidad de captación del radiofármaco se cuantifica habitualmente mediante el índice SUV (Standard Uptake Value) utilizando la siguiente fórmula:

$$
\begin{gathered}
\mathrm{SUV}=\text { concentración del VOI }(\mathrm{Bq} / \mathrm{g}) \mathrm{x} \\
\text { peso paciente }(\mathrm{g}) / \text { dosis inyectada }(\mathrm{Bq})
\end{gathered}
$$




\section{EQUIPOS PET/TC}

El primer prototipo de equipo híbrido PET/TC fue desarrollado en la Universidad de Pittsburg en 1998 y el equipo híbrido PET/TC estaba disponible comercialmente en $2001^{5}$. La motivación que impulsó el diseño de este equipo fue la obtención de imágenes clínicas tanto de tomografía por emisión de positrones como de tomografía computarizada, alineadas con precisión, en un mismo equipo. El equipo PET/TC, combinando las dos modalidades de imagen, es una evolución de la tecnología de imagen existente, integrando dos técnicas que han progresado históricamente por separado, aunque la PET debe mejorar en resolución espacial y rapidez de adquisición. Los equipos PET/TC combinan un TC multidetector helicoidal que proporciona la información anatómica (localizado proximalmente en el estativo) con un tomógrafo PET dedicado que proporciona la información metabólica (localizado distalmente en el estativo). La fusión automática de ambas modalidades se realiza en una estación de trabajo dedicada.

La PET es una modalidad de imagen funcional excelente, pero tiene limitaciones inherentes a la resolución espacial y a la falta de especificidad del radiofármaco FDG para lesiones tumorales malignas. La PET no proporciona información anatómica suficiente para la localización exacta de las lesiones. El desarrollo de los equipos híbridos PET/ $\mathrm{TC}$ ha resuelto estas limitaciones: con una exploración única al paciente se logra la fusión de las imágenes metabólicas de la PET con las imágenes morfoanatómicas de la TC $\mathrm{y}$, por tanto, se mejora el rendimiento diagnóstico de la PET/TC con relación a los estudios realizados sólo con PET o TC ${ }^{6}$. En los pacientes con tumores de cabeza y cuello esta técnica es de mayor valor incluso que en el resto de patologías dada la complejidad de la anatomía y la presencia de numerosas estructuras normales con intensidad de captación fisiológica variable de FDG.

\section{Protocolos de obtención de imágenes PET/TC}

Los equipos híbridos PET/TC permiten la adquisición de los estudios metabólico y anatómico en el mismo aparato, en el que se combinan los componentes de los equipos PET y TC en un mismo estativo. Con esta combinación, los estudios se adquieren de modo secuencial, con una diferencia mínima de tiempo entre ambos; no se modifica el perfil de la camilla y no es preciso reposicionar al paciente. El protocolo de un estudio con un equipo PET/ TC es parecido al protocolo PET estándar, siendo la mayor diferencia la sustitución del estudio de transmisión con fuentes radiactivas $\left({ }^{68} \mathrm{Ge} \mathrm{o}{ }^{137} \mathrm{Cs}\right)$ por otro empleando los rayos X de la TC. Es conveniente considerar las distintas fases del protocolo: preparación del paciente, posicionamiento del mismo en el tomógrafo, realización de un topograma, adquisición de los estudios TC y de emisión de la PET, reconstrucción y análisis de las imágenes corregidas por atenuación y sin corregir.

La FDG se administra de forma intravenosa, dejando transcurrir 50-60 minutos desde la inyección hasta la obtención de las imágenes, para permitir alcanzar una incorporación estable del radiofármaco en los tejidos. La posición del paciente en la camilla debe ser confortable, para minimizar movimientos involuntarios durante la realización de los estudios, que llevarían a un error en el corregistro de las imágenes PET y TC, además de una incorrecta asignación de la atenuación calculada a partir de la TC. Para evitar estos movimientos se pueden utilizar accesorios o dispositivos de inmovilización.

En la PET/TC no se utilizan contrastes radiológicos como técnica de rutina, aunque existe una tendencia creciente a utilizarlos, de forma que el estudio de TC puede utilizarse para el diagnóstico. Cuando se utiliza el protocolo con contraste intravenoso, un especialista en medicina nuclear interpreta la exploración PET y un radiólogo interpreta la exploración TC de cabeza y cuello. Esta técnica puede proporcionar en una sola exploración del paciente la información necesaria para:

- La delineación precisa de los márgenes del tumor y de las metástasis, tanto ganglionares como a distancia. 
- La evaluación de la extensión de la lesión por fuera de los espacios mucosos de la faringe o laringe.

- La detección de la extensión extralaríngea de tumores laríngeos.

- La evaluación de la invasión tumoral de estructuras adyacentes, vasos, hueso y cartílago, fascia prevertebral, crecimiento perineural, extensión hacia la base del cráneo y la extensión extracapsular de la infiltración ganglionar.

No obstante, son necesarios estudios prospectivos para determinar el impacto diagnóstico adicional de la PET/TC realizada con contraste intravenoso en la estadificación inicial y en el seguimiento de los pacientes.

\section{Indicaciones}

La PET-FDG está aceptada en la detección de tumores de origen desconocido evidenciados por adenopatías laterocervicales y en la estadificación inicial de pa- cientes con tumores malignos de cabeza y cuello. Una estadificación precisa es fundamental, no sólo para el tratamiento adecuado de los pacientes, sino también por sus implicaciones pronósticas y porque permite evitar terapias agresivas o inútiles en pacientes con enfermedad diseminada. La PET-FDG también está aceptada en la estadificación inicial, con especial contribución en la estadificación ganglionar y en la detección de metástasis a distancia y segundos primarios (Tabla 1). Con la tendencia hacia tratamientos de preservación de órgano, la evaluación de la respuesta al tratamiento es otra indicación aceptada que permite evitar cirugías innecesarias en ausencia de enfermedad residual tras tratamientos de radioterapia con o sin quimioterapia. La PET-FDG también tiene indicación clínica en el seguimiento de los pacientes tratados, tanto para la detección de recidiva como de enfermedad metastásica. Además se está investigando la PET/TC en la planificación de radioterapia y se ha observado utilidad para la identificación de segundos primarios en el seguimiento.

Tabla 1. Indicaciones clínicas aceptadas de la PET-FDG en tumores de cabeza y cuello.

\begin{tabular}{l}
\hline Indicaciones clínicas \\
\hline Detección del tumor de origen desconocido evidenciado por adenopatía laterocervical \\
Estadificación inicial \\
Evaluación de respuesta al tratamiento \\
Detección de recidiva \\
\hline
\end{tabular}

\section{ESTADIFICACIÓN INICIAL}

La delimitación precisa del tumor primario y de la enfermedad metastásica ganglionar es fundamental en la estadificación inicial para un manejo terapéutico óptimo. La resecabilidad depende de la extensión tumoral que habitualmente se valora con las TDC. La PET-FDG tiene mayor precisión que las TDC en la estadificación y cambia el estadio inicial hasta en un $34 \%$ de los pacientes, con impacto clínico debido a las modificaciones en el tratamiento ${ }^{7}$. Así, al cambiar principalmente la estadificación ganglionar produce modificaciones en el abordaje terapéutico. Por otro lado, la detección de metástasis a distancia determina un cambio de tratamiento con intención curativa a paliativa. La PET-FDG también puede ser de utilidad en determinadas situaciones clínicas como la detección de un tumores primarios síncronos ${ }^{8}$.

\section{Tumor primario}

Aunque numerosas publicaciones han mostrado que la PET-FDG es al menos tan 
sensible como las TDC en la detección del tumor primario, tanto la PET con fuentes de germanio como la PET/TC sin contraste tienen impacto mínimo en la estadificación $\mathrm{T}$ de los tumores de cabeza y cuello debido a la limitación de la resolución anatómica. La exploración clínica y las TDC proporcionan información de mayor definición para delimitar la extensión local y la infiltración de estructuras adyacentes, con la precisión anatómica necesaria para el abordaje terapéutico. La PET-FDG tiene limitaciones para detectar tumores pequeños estadio T1, tumores de extensión superficial, tumores con extensión perineural y adenocarcinomas en glándulas salivales.

\section{Metástasis ganglionares}

Es fundamental disponer de la máxima información en cuanto a las características de la enfermedad ganglionar, principalmente en su uni o bilateralidad así como en la existencia de rotura capsular y extensión a estructuras anatómicas adyacentes. La exploración clínica puede dar falsos negativos en $30-40 \%$ y las TDC en $20-30 \%$ de los ganglios de tamaño inferior a $1 \mathrm{~cm}$. La PETFDG es superior a las TDC en la detección de infiltración ganglionar tumoral, tanto en sensibilidad como en especificidad, ya que el criterio diagnóstico no depende del tamaño de los ganglios sino del aumento del metabolismo de FDG $^{9}$. Además los equipos híbridos PET/TC permiten la localización exacta de los ganglios afectos y ayudan a caracterizar la naturaleza de ganglios menores de $15 \mathrm{~mm}$ detectados en las TDC. Debe tenerse en cuenta que debido a la posibilidad de falsos diagnósticos positivos, se debe realizar confirmación histológica de los hallazgos. Además, en pacientes con exploración clínica positiva en el cuello la PET-FDG puede detectar más enfermedad o enfermedad contralateral que determina cambios en el tratamiento de los pacientes. Se aconseja realizar un estudio PET/TC en la estadificación inicial que servirá de referencia en el seguimiento posterior a la terapia. No obstante, el valor predictivo negativo de la PET-FDG está en torno al 85\%. $\mathrm{Y}$ en trabajos prospectivos publicados re- cientemente en tumores de cavidad oral, un 15-33\% de pacientes con exploración clínica y PET-FDG negativas en cuello tenían enfermedad ganglionar oculta ${ }^{10}$. También la biopsia selectiva del ganglio centinela ha puesto de manifiesto la limitación de la PET-FDG en la detección de las metástasis ganglionares de menor tamaño, por debajo de $5 \mathrm{~mm}^{11}$. Por otro lado, las metástasis ganglionares con componente quístico o necrótico captan menos e incluso pueden no captar FDG, siendo otra causa de falsos diagnósticos negativos.

\section{Metástasis a distancia y segundos tumores primarios}

La incidencia de metástasis a distancia en el diagnóstico inicial varía de $2 \%$ a $18 \%$, según las series, pero es considerada en general bastante baja, por lo que no se realiza de forma rutinaria cribado de metástasis a distancia. Sin embargo, los pacientes con tumores grandes, con estadio T elevado, o con infiltración metastásica ganglionar, tienen mayor riesgo de metástasis a distancia en el diagnóstico inicial, fundamentalmente en pulmón, hueso e hígado. También tienen mayor riesgo de segundos primarios en pulmón y en las vías aérea y digestiva superiores, sobre todo los pacientes con historia de consumo elevado de tabaco o alcohol. La PET-FDG es una técnica de mayor sensibilidad que las TDC y gracias a la PET/ TC es posible una localización precisa de las lesiones tumorales hipercaptantes. Hay autores que afirman que, en pacientes seleccionados por alto riesgo de desarrollar metástasis a distancia, la PET/TC con FDG puede sustituir a las TDC, ya que presenta mayor sensibilidad y permite explorar en un solo estudio desde la base del cráneo hasta la pelvis ${ }^{12}$. Al detectar enfermedad no identificada por las TDC hasta en un $27 \%$ de los pacientes, evita tratamientos agresivos inapropiados o inútiles. En los pacientes con tumores de cabeza y cuello tiene impacto clínico en el manejo terapéutico y mejora la estratificación pronóstica ${ }^{13}$. Su mejor rendimiento es debido a que permite realizar un estudio de cuerpo entero en 
una sola exploración; es superior a las TDC en la detección de enfermedad metastásica de pequeño volumen tumoral, también en la detección de enfermedad ósea y detecta enfermedad ganglionar fuera de las regiones exploradas habitualmente (mediastino y axila).

La PET-FDG se utiliza en la valoración no invasiva de los nódulos pulmonares de 1-1,5 cm indeterminados por TDC, debido a su elevado valor predictivo positivo. En nódulos pulmonares de tamaño mayor a $1 \mathrm{~cm}$ el valor predictivo negativo es menor. Por otro lado, existen falsos diagnósticos positivos en patología inflamatoria. La experiencia en la interpretación en el contexto clínico es muy importante: los nódulos pulmonares en pacientes con enfermedad localmente avanzada o con metástasis ganglionares localizadas en cadenas ganglionares inferiores (nivel IV, V y región supraclavicular) tienen mayor probabilidad de ser metastásicos. Por el contrario, pacientes de edad avanzada y hábito tabáquico sin enfermedad ganglionar o con escasa enfermedad localizada en cadenas ganglionares superiores, tienen mayor probabilidad de tener un segundo primario.

\section{Re-estadificación en pacientes tratados}

El tratamiento inicial, tanto con cirugía como con radioterapia, provoca cambios en el tejido sano con edema y fibrosis y estas distorsiones dificultan la identificación de enfermedad residual o recidiva tanto en la exploración física como en las TDC. El tratamiento con quimio-radioterapia es una alternativa no quirúrgica que se aplica en tumores irresecables, en protocolos de preservación de órgano en pacientes con tumores de laringe, orofaringe o hipofaringe, con intención de evitar la resección quirúrgica y en situaciones en las que se ha demostrado que se logra el mismo control loco-regional o mejor que con tratamiento quirúrgico. Una de las áreas de mayor impacto clínico de la PET es la re-estadificación de pacientes con enfermedad metastásica ganglionar estadio
N2-N3 tratados con radioterapia ya que, si existe respuesta metabólica completa, la observación puede ser una alternativa a la linfadenectomía planificada. Ello es debido al elevado valor predictivo negativo y alta especificidad para excluir enfermedad residual locorregional ${ }^{14}$. En pacientes con cáncer de laringe, orofaringe o hipofaringe la re-estadificación tras los protocolos de preservación de órgano también es fundamental. La PET-FDG se está aplicando de forma creciente para evaluar la respuesta metabólica al tratamiento de quimio-radioterapia por dos razones. Primera, porque permite valorar los cambios en las características morfofuncionales en una sola exploración. Segunda, porque al distinguir el tejido cicatricial del tejido tumoral, permite identificar los pacientes con enfermedad residual susceptible de rescate quirúrgico con mayor precisión que las TDC. Los trabajos publicados han demostrado una sensibilidad elevada (84-100\%), pero con una especificidad moderada (61-93\%). Es necesario tener en cuenta que la PET-FDG puede dar falsos diagnósticos positivos tras el tratamiento con radioterapia, incluso con valores de captación SUV elevados, debido a la reacción inflamatoria tanto en tejido linfoide como en glándulas salivales, músculo y partes blandas adyacentes. Por ello es importante estar familiarizado con los cambios producidos por el tratamiento que pueden provocar captación de FDG. Para evitar falsos diagnósticos positivos y obtener un elevado valor predictivo negativo se aconseja realizar la PET tras un mínimo de 2 meses según algunos autores y 3 meses en la mayoría de las series tras finalizar tratamiento radio/quimioterápico (Tabla 2). Estos autores afirman que la PET tiene un elevado valor predictivo negativo, superior al $95 \%$ con una mediana de 15 semanas tras el tratamiento. Cuando la PET es positiva pasados 3 meses del fin de la radioterapia se recomienda correlacionar los hallazgos con la información de la exploración física y de la TC y confirmar mediante biopsia. Si la biopsia es negativa se aconseja realizar la PET pasados 5 meses del fin de la radioterapia. Si el valor de captación SUV obtenido a los 5 meses dismi- 
nuye respecto al obtenido a los 3 meses, la probabilidad de recidiva es prácticamente inexistente. Pero si el valor SUV no cambia o aumenta, se recomienda repetir la biop- sia excepto en situaciones clínicas que expliquen dicha evolución metabólica, como presencia de úlcera, infección, fístula o radionecrosis.

Tabla 2. Estudios PET FDG en la detección de enfermedad residual con diferentes intervalos tras quimio/radioterapia.

\begin{tabular}{|c|c|c|c|c|c|c|c|}
\hline Autores & Año & $\mathbf{N}$ & Sensibilidad & Especificidad & VPP & VPN & Intervalo \\
\hline Wong y coll ${ }^{15}$ & 2002 & 143 & $\begin{array}{l}\text { Local: } 97 \\
\text { Regional: } 92\end{array}$ & $\begin{array}{l}\text { Local: } 79 \\
\text { Regional: } 95\end{array}$ & $\begin{array}{l}54 \\
77\end{array}$ & $\begin{array}{l}99 \\
99\end{array}$ & 7 meses \\
\hline Yao y col ${ }^{16}$ & 2005 & 85 & $\begin{array}{l}\text { Local: } 86 \\
\text { Regional: } 100\end{array}$ & $\begin{array}{l}\text { Local: } 90 \\
\text { Regional: } 96\end{array}$ & $\begin{array}{l}55 \\
78\end{array}$ & $\begin{array}{r}98 \\
100\end{array}$ & 3-5 meses \\
\hline Porceddu y col ${ }^{17}$ & 2005 & 39 & Regional: 88 & Regional: 100 & 100 & 97 & 12 semanas \\
\hline Ryan y col ${ }^{18}$ & 2005 & 103 & $\begin{array}{l}\text { Local: } 87 \\
\text { Regional: } 79\end{array}$ & $\begin{array}{l}\text { Local: } 89 \\
\text { Regional: } 95\end{array}$ & $\begin{array}{l}54 \\
75\end{array}$ & $\begin{array}{l}98 \\
96\end{array}$ & 8 semanas \\
\hline Andrade y col ${ }^{19}$ & 2006 & 28 & Local: 77 & Local: 93 & 91 & 82 & 8 semanas \\
\hline Chen y col' ${ }^{20}$ & 2006 & 30 & $\begin{array}{l}\text { Local: } 50 \\
\text { Regional: } 100\end{array}$ & $\begin{array}{l}\text { Local: } 85 \\
\text { Regional: } 70\end{array}$ & $\begin{array}{l}20 \\
36\end{array}$ & $\begin{array}{r}95 \\
100\end{array}$ & 7 semanas \\
\hline Kim SY y col' ${ }^{21}$ & 2007 & 97 & $\begin{array}{l}\text { Local: } 83 \\
\text { Regional: } 100\end{array}$ & $\begin{array}{l}\text { Local: } 92 \\
\text { Regional: } 99\end{array}$ & $\begin{array}{l}59 \\
83\end{array}$ & $\begin{array}{r}98 \\
100\end{array}$ & 4 semanas \\
\hline
\end{tabular}

\section{Seguimiento}

En los pacientes tratados por tumores de cabeza y cuello es recomendable un seguimiento exhaustivo, sobre todo en los primeros 12-15 meses, ya que es en este período cuando se producen la mayor parte de las recidivas, tanto locales como en pulmón, hueso e hígado. La información de la PET tiene valor pronóstico en cuanto a la supervivencia de los pacientes ya que el valor de captación SUV en la recidiva metabólica muestra una buena correlación tanto con la supervivencia global como con la supervivencia libre de enfermedad ${ }^{15}$. En pacientes tratados con quimio-radioterapia con respuesta completa clínica y radiológica, es aconsejable realizar el estudio PET/ TC a los 3 meses. Si hay focos hipercaptantes en la PET, se aconseja realizar la PET pasados 5 meses del fin de la radioterapia para evitar falsos diagnósticos positivos por inflamación post-radiación. Se recomienda correlacionar siempre los hallazgos con la información de la exploración física y de las TDC y obtener confirmación histológica previa a nuevas actuaciones terapéuticas. En caso de objetivar respuesta metabólica completa a los 5 meses, es recomendable el seguimiento regular del paciente con exploración física y TDC. En determinadas localizaciones como la nasofaringe, tumores nasosinusales y de base de cráneo, puede ser útil complementar la información con RM por su mayor sensibilidad respecto a la TC.

\section{NUEVOS RADIOFÁRMACOS PET}

La PET es una modalidad de imagen que puede detectar positrones de una amplia variedad de isótopos además del flúor-18, incluyendo el carbono-11, oxígeno-15 y nitrógeno-13. Además de la FDG se emplean otros radiofármacos para la detección selectiva de tejido tumoral. Por ejemplo, la L-metil- ${ }^{11} \mathrm{C}$-metionina es un aminoácido que accede al interior de las células mediante sistemas de transporte y se incorpora a proteínas y metabolitos intermedios a diferentes niveles. Se ha utilizado para es- 
tudiar el metabolismo proteico con mayor especificidad que la FDG. La flúor-18-fluorodesoxi-L-timidina (FLT) también ha generado interés por su capacidad para valorar la síntesis de DNA, mostrando correlación con la proliferación tumoral celular. La FLT es fosforilada por la timidin-kinasa y atrapada en las células en la vía de la síntesis de DNA. La imagen PET de la proliferación celular puede aportar nuevos conocimientos de la biología tumoral. Otro factor a tener en cuenta en los tumores malignos es la hipoxia, ya que es frecuente y se asocia con progresión tumoral y resistencia a la radioterapia. Se están evaluando radiofármacos como el flúor-18-fluoromisonidazol ya que se acumula de forma selectiva en el tejido hipóxico pero apenas muestra captación en tejidos con oxigenación normal. Se ha empleado en diversos trabajos para la caracterización biológica de los tumores de cabeza y cuello previa al tratamiento con radioterapia, proporcionando información pronóstica. Esta caracterización permite una terapia molecular más específica, junto con la radioterapia, en pacientes con hipoxia tumoral identificada en PET.

No obstante, aunque estos radiofármacos han mostrado resultados prometedores en tumores de cabeza y cuello y pueden tener impacto clínico en el futuro, dependen de trabajos de investigación más amplios que permitan validar su aplicación clínica.

\section{Planificación de radioterapia}

La radioterapia es un tratamiento fundamental en los tumores de cabeza y cuello (TCC), ya sea de forma exclusiva o en combinación con quimioterapia y/o cirugía. Se calcula que un $80 \%$ de los pacientes con esta patología recibirán radioterapia como parte de su tratamiento oncológico. El éxito del tratamiento depende en gran medida de su correcto diseño y aplicación. La planificación de la radioterapia en los tumores de cabeza y cuello es un proceso complejo cuyo primer paso es la delimitación de los volúmenes a irradiar (CTVs) en los cortes del TC efectuados en la posición de tratamiento, usualmente en decúbito supino con el cuello en hiperextensión e inmovili- zación de cabeza y hombros con máscaras de termoplásticos. Aunque el papel del PET en el diagnóstico de extensión e incluso en el seguimiento y detección precoz de la recidiva está bien establecido, el impacto real de una mejor planificación del tratamiento radioterápico gracias a que el PET/ TC ayuda a delimitar mejor las áreas de afectación tumoral que el TC en el control local de la enfermedad y, por tanto, en la supervivencia, aún no ha sido demostrado.

Los equipos híbridos PET/TC proporcionan la información funcional y anatómica en una sola exploración, lo cual supone una innovación en la planificación de la radioterapia con técnicas de imagen funcional. La aportación de la PET/TC a la delineación espacial precisa de los volúmenes a irradiar es especialmente importante con modalidades como la radioterapia de intensidad modulada o la radiocirugía estereotáxica, ambas utilizadas en el tratamiento de pacientes con cáncer de cabeza y cuello. Para la realización de la PET/ TC en esta indicación se utiliza una mesa de exploración plana, similar a la utilizada durante el tratamiento. Los pacientes son inmovilizados en la misma posición que van a mantener durante el tratamiento radioterápico mediante dispositivos especiales, incluyendo máscaras termoplásticas y se recurre a láseres externos al tomógrafo para optimizar el registro de las imágenes y su aplicación en la planificación del tratamiento. Una vez adquiridas y procesadas las imágenes del PET/TC, éstas deben ser transferidas al sistema de planificación de radioterapia para su utilización en el contorneo de los volúmenes a irradiar.

\section{PET-TC en la delimitación de volúmenes (CTV) en los carcinomas de cabeza y cuello}

La PET/TC contribuye a una definición de los volúmenes a tratar y del cálculo de la dosis con mayor precisión que la $\mathrm{TC}^{22}$. No obstante, es menos sensible que la RM en la identificación de infiltración de médula ósea adyacente y en la extensión perineural. La evidencia publicada pone de mani- 
fiesto un aumento del GTV (Gross Tumor Volume) con PET/TC versus TC hasta en un $20 \%$ de los $\operatorname{casos}^{23}$. A pesar de que el uso del PET, y sobre todo del PET-TC para la delineación de los CTVs es cada vez más popular, el manejo estandarizado de dichas imágenes para una correcta delineación de las estructuras a irradiar aún no ha sido resuelto con solvencia. En efecto, los estudios pioneros en dicha utilización se basaban en criterios tan subjetivos como el de contorneo de volúmenes por especialistas tanto de Medicina Nuclear como de Oncología Radioterápica, bien experimentados en el manejo de dicha técnica ${ }^{24,25}$. En este sentido, otros autores han desarrollado otros métodos más objetivos y reproducibles como la utilización de un porcentaje fijo de actividad (por ejemplo, un porcentaje del SUV máximo en el tumor, para delinear el margen del mismo). Paulino, usando un porcentaje fijo del $50 \%$ del SUV del el tumor, demostró que los CTVs delineados eran mayores que los delineados con el TC en el $25 \%$ de los $\operatorname{casos}^{26}$. Los resultadas deben tomarse con precaución dado el porcentaje del SUV seleccionado de forma arbitraria en estos estudios, por lo que sus resultados deben ser aún validados. Aunque los métodos fijos son más reproducibles, no son del todo adecuados para delimitar los límites de los tumores con precisión, por lo que se ha intentado utilizar otros métodos adaptativos, como la intensidad de la señal en relación al "fondo" ${ }^{23}$. Con el fin de unificar criterios, se reunieron en Viena en julio del 2006 un grupo de expertos en PET/RT. Sus conclusiones han sido recientemente publicadas en la revista Radiotherapy and Oncology en el año $2009^{27}$.

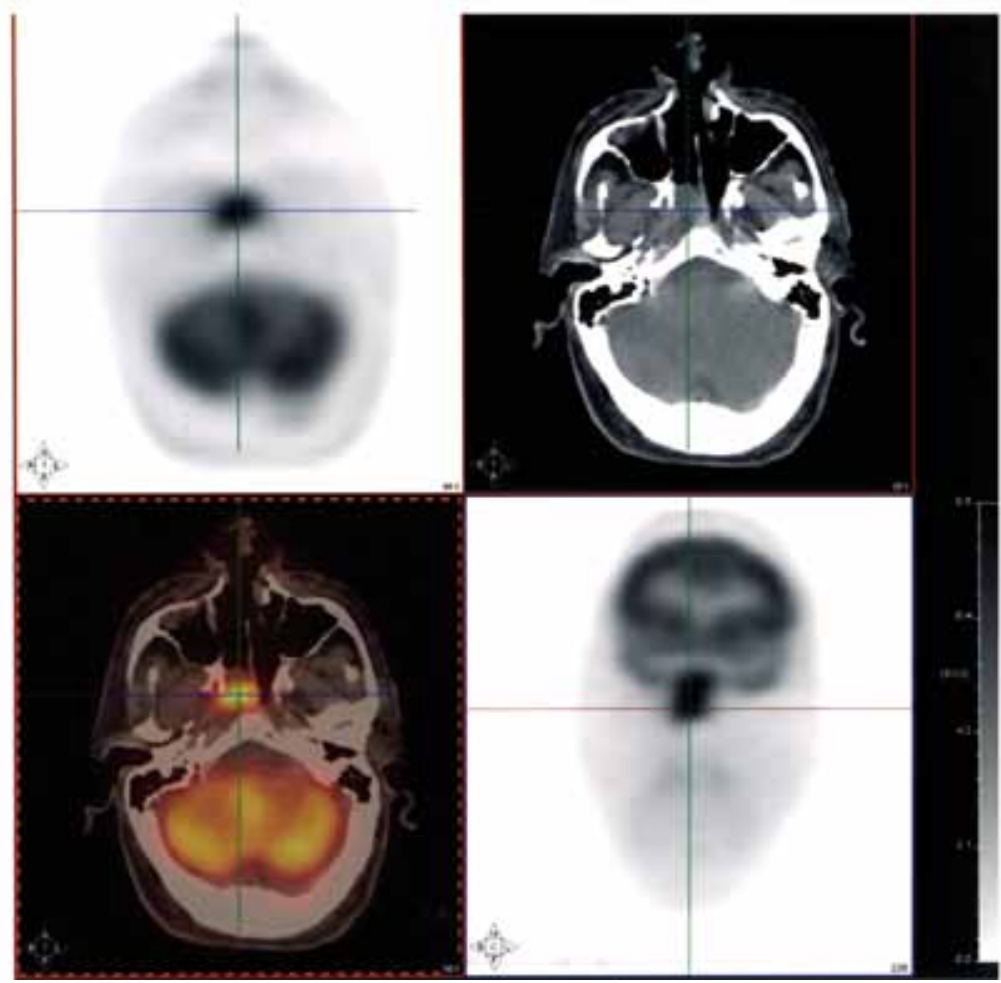

Figura 1. TC y PET en el momento de la recidiva. 
Un caso concreto en el que la planificación con PET/CT puede ser especialmente útil se trata de los casos de recidivas locales en áreas ya irradiadas, en los que las imágenes del CT o RM pueden no ser muy adecuadas para delimitar los márgenes de la tumoración con la precisión necesaria para cubrir bien el área tumoral a la vez que proteger las estructuras sanas próximas. Un ejemplo puede verse en la figuras
1 y 2 , que corresponden a un paciente con una recidiva local a los 2 años de recibir quimio-radioterapia por un tumor de cavum localmente avanzado, en el que se planificó la radioterapia basada en el TC pero asegurando una buena cobertura del área captante en el PET. El paciente completó el tratamiento sin complicaciones y permanece en respuesta completa a los 2 años de la reirradiación.

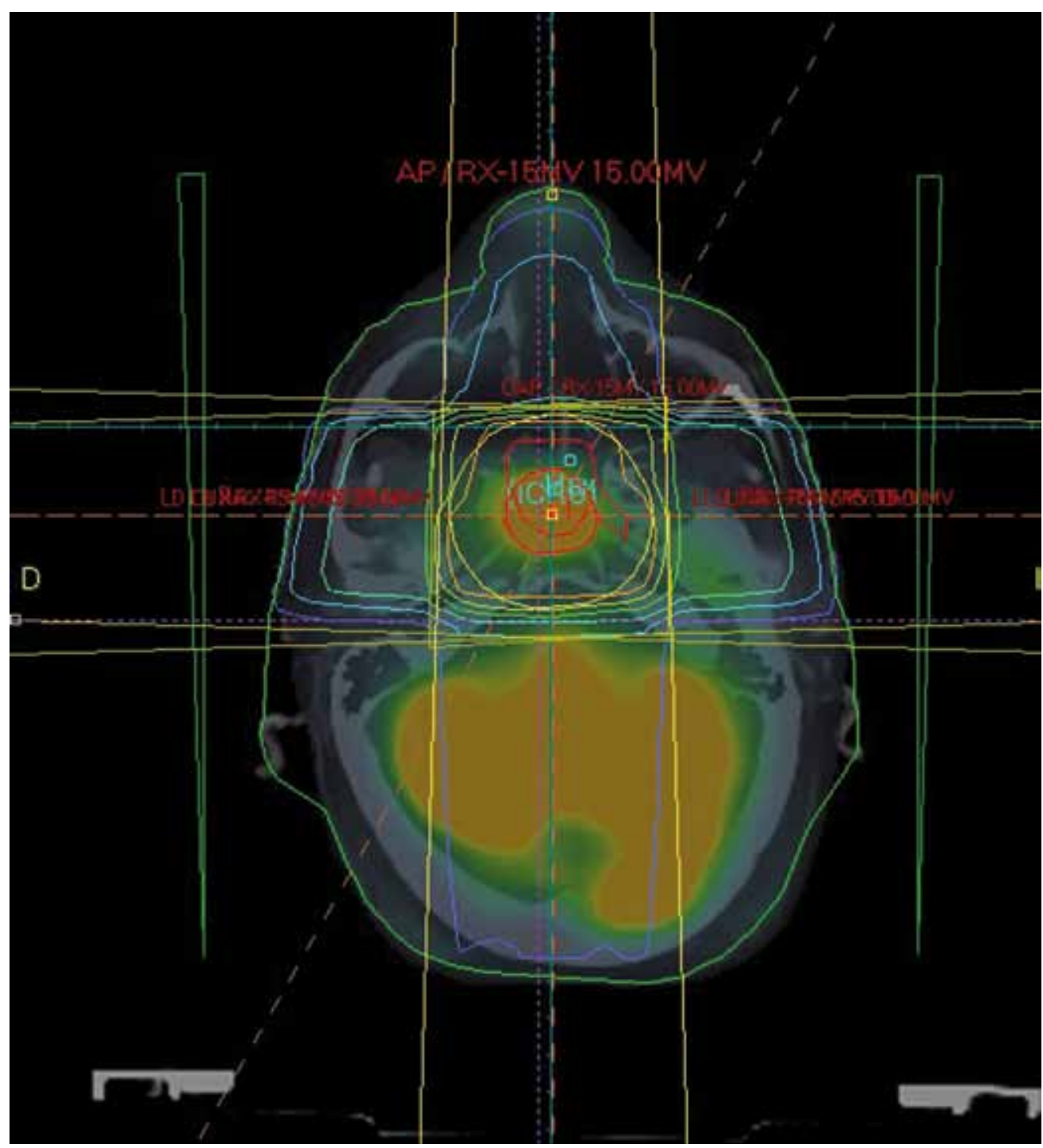

Figura 2. Campos de irradiación empleados en este paciente. Puede observarse que el campo abarca ampliamente la zona captante del PET. 
En resumen, podemos concluir que el PET, y en especial el PET/CT está revolucionando el manejo de los tumores de cabeza y cuello, especialmente de los localmente avanzados que son los más frecuentes. Ahora más que nunca son necesarios estudios clínicos diseñados para cuantificar el impacto del PET/TC en la planificación del tratamiento radioterápico de los pacientes con carcinomas de cabeza y cuello tratados con radioterapia radical. Dicho impacto se debería trasladar en unas mayores tasas de respuesta y control local.

\section{BIBLIOGRAFÍA}

1. Ardanaz E, Moreno MC, Pérez de Rada MC, EzPonda C, Floristan Y, Navaridas N et al. Incidencia y mortalidad por cáncer en Navarra, 1998-2002: evolución en los últimos 30 años. An Sist Sanit Navar 2007; 30: 245-270.

2. Vokes, EE, Weichselbaum RR, Lippman SM, Hong WK. Head and neck cancer. N Engl J Med 1993; 328: 184-194.

3. Porceddu SV, Burmeister BH, Hicks RJ. Role of functional imaging in head and neck squamous cell carcinoma: fluorodeoxyglucose positron emission tomography and beyond. Hematol Oncol Clin North Am 2008; 22: 12211238.

4. Schöder H, Yeung HW. Positron emission imaging of head and neck cancer, including thyroid carcinoma. Semin Nucl Med 2004; 34 : 180-197.

5. Beyer T, Townsend DW, Brun T, Kinahan PE, Charron M, Roddy R et al. A combined PET/ CT scanner for clinical oncology. J Nucl Med 2000; 41: 1369-1379.

6. Branstetter BF 4th, Blodgett TM, Zimmer LA, SNYDERman CH, JohnSON JT, Raman S et al. Head and neck malignancy: is PET/CT more accurate than PET or CT alone? Radiology 2005; 235: 580-586.

7. Connell CA, Corry J, Milner AD, Hogg A, Hicks RJ, RIschin D et al. Clinical impact of, and prognostic stratification by, F-18FDG-PET/ CT in head and neck mucosal squamous cell carcinoma. Head Neck 2007; 29: 986-995.

8. KIm SY, Roh JL, Yeo NK, KIM JS, LeE JH, ChO SH et al. Combined 18F-fluorodeoxyglucose-positron emission tomography and computed tomography as a primary screening method for detecting second primary cancers and distant metastases in patients with head and neck cancer. Ann Oncol 2007; 18: 1698-1703.

9. Jeong HS, Baek CH, Son YI, Ki Chung M, Kyung Lee D, Young Chol J et al. Use of integrated 18F-FDG PET/CT to improve the accuracy of initial cervical nodal evaluation in patients with head and neck squamous cell carcinoma. Head Neck 2007; 29: 203-210.

10. Schöder H, Carlson DL, Kraus DH, Stambuk HE, GöNEN M, ERDI YE et al. 18F-FDG PET/CT for detecting nodal metastases in patients with oral cancer staged N0 by clinical examination and CT/MRI. J Nucl Med 2006; 47: 755762.

11. Hyde NC, Prvulovich E, Newman L, Waddington WA, Visvikis D, Ell P. A new approach to pretreatment assessment of the $\mathrm{N} 0$ neck in oral squamous cell carcinoma: the role of sentinel node biopsy and positron emission tomography. Oral Oncol 2003; 39: 350-360.

12. NG SH, Chan SC, Yen TC, Chang JT, Liao CT, Ko SF et al. Staging of untreated nasopharyngeal carcinoma with PET/CT: comparison with conventional imaging work-up. Eur J Nucl Med Mol Imaging 2009; 36: 12-22.

13. Scott AM, Gunawardana DH, Bartholomeusz D, RAMSHAW JE, LIN P. PET changes management and improves prognostic stratification in patients with head and neck cancer: results of a multicenter prospective study. J Nucl Med 2008; 49: 1593-1600.

14. Ong SC, Schöder H, Lee NY, Patel SG, Carlson D, FURY M et al. Clinical utility of 18F-FDG PET/ $\mathrm{CT}$ in assessing the neck after concurrent chemoradiotherapy for locoregional advanced head and neck cancer. J Nucl Med 2008; 49: 532-540.

15. Wong RJ, Lin DT, Schöder H, Patel SG, Gonen M, Wolden S et al. Diagnostic and prognostic value of [(18)F] fluorodeoxyglucose positron emission tomography for recurrent head and neck squamous cell carcinoma. J Clin Oncol 2002; 20: 4199-4208.

16. Yao M, Smith RB, Graham MM, Hoffman HT, TAN H, FunK GF et al. Rhe role of FDG PET in management of neck metastasis from headand-neck cancer after definitive radiation treatment. Int J Radiat Oncol Biol Phys 2005; 63: 991-999.

17. Porceddu SV, Jarmolowski E, Hicks RJ, Ware R, WEIH L, RISCHIN D et al. Utility of positron emission tomography for the detection of disease in residual neck nodes after (chemo) radiotherapy in head and neck cancer. Head Neck 2005; 27 : 175-181. 
18. Ryan WR, FeE WE JR, Le QT, PINTo HA. Positronemission tomography for surveillance of head and neck cancer. Laryngoscope 2005; 115: 645-650.

19. Andrade RS, Heron DE, Degirmenci B, Filho PA, Branstetter BF, Seethala RR et al. Posttreatment assessment of response using FDG-PET/CT for patients treated with definitive radiation therapy for head and neck cancers. Int J Radiat Oncol Biol Phys 2006; 65: 1315-1322.

20. Chen AY, Vilaseca I, Hudgins PA, Schuster D, HALKAR R. PET-CT vs contrast-enhanced CT: what is the role for each after chemoradiation for advanced oropharyngeal cancer? Head Neck 2006; 28: 487-495.

21. KIm SY, LeE SW, Nam SY, Im KC, KIm JS, OH SJ et al. The feasibility of 18F-FDG PET scans 1 month after completing radiotherapy of squamous cell carcinoma of the head and neck. J Nucl Med 2007; 48: 373-378.

22. Ahn PH, GARG MK. Positron emission tomography/computed tomography for target delineation in head and neck cancers. Semin Nucl Med 2008; 38: 141-148.

23. Daisne JF, Duprez T, Weynand B, Lonneux M, HaMOIR M, REYChLER $\mathrm{H}$, et al. Tumor volume in pharyngolaryngeal squamous cell carcinoma: comparison at CT, MR imaging, and FDG PET and validation with surgical specimen. Radiology 2004; 233: 93-100.

24. Ciernik IF, Dizendorf E, Baumert BG, Reiner B, Burger C, DAvis JB et al. Radiation treatment planning with an integrated positron emission and computer tomography (PET/CT): a feasibility study. Int J Radiat Oncol Biol Phys 2003; 57: 853-863.

25. Nishioka T, Shiga T, Shirato H, Tsukamoto E, TsuCHIYA K, KATO $\mathrm{T}$ et al. Image fusion between 18FDG-PET and MRI/CT for radiotherapy planning of oropharyngeal and nasopharyngeal carcinomas. Int J Radiat Oncol Biol Phys 2002; 53: 1051-1057.

26. Paulino AC, Koshy M, Howell R, Schuster D, DAvis LW. Comparison of CT- and FDG-PET defined gross tumor volume in intensity modulated radiotherapy for head and neck cancer. Int J Radiat Oncol Biol Phys 2005; 61: 1385-1392.

27. Macmanus M, Nestle U, Rosenzweig KE, Carrio I, Messa C, Belohlavek O et al. Use of PET/CT for radiation therapy planning: IAEA expert report 2006-2007. Radiother Oncol 2009; 1: 85-94. 